\title{
Generic Computation of bulletin boards into Geometric Kernels
}

\author{
Mehdi Baba-ali* \\ Signal Image Communications Lab \\ University of Poitiers.
}

\author{
David Marcheix ${ }^{\dagger}$ \\ LISI Lab/ ENSMA.
}

Yves Bertrand $\AA^{\S}$

Signal Image Communications Lab

University of Poitiers.

\author{
Xavier Skapin $\ddagger$ \\ Signal Image Communications Lab \\ University of Poitiers.
}

\begin{abstract}
Nowadays, many commercial CAD systems are built on proprietary geometric kernels which provide an API containing a set of high level geometric operations (boolean operations, slot, chamfering, etc). Because of their complexity, these operations can generate important modifications on topological cells (vertices, edges, faces, volumes, etc.) of the objects. At the same time, many of these kernels need to know precisely what has occurred to each topological cell belonging to objects given or resulting from a previous high level geometric operation. At the end of each operation, the geometric kernel must provide a bulletin board describing cells' evolution through a list of events (split, merge, creation, deletion).

Most commercial geometric kernels use B-Rep structures and provide methods enabling the developer of a CAD system to retrieve a number of events that occurred on cells. These kernels have their own scheme for detecting events, based on their own taxonomy of situations, heuristics and evolution rules. Little is known of their details, which are proprietary information, let alone of the underlying theory, if any. Generally, for example, the detected events are not generic for all cells' dimensions. This lack of underlying theory limits the possibility to extend the use of these kernels to new domains of investigation.

In this paper, we propose a generic model that enables to create a bulletin board. This bulletin board will contain the complete list of events having occurred on cells of any dimension, and that belong to any topological model. The genericity of this model and the completeness in all dimensions of this list are based on the use of four elementary mechanisms (split_elem, merge_elem, crea_elem, del_elem). They are defined independently of the topological model, and allow the generation of the bulletin board, whatever the geometric operation. This model has been implemented using the geometric kernel of the modeler Moka, based on generalized maps.
\end{abstract}

Keywords: Bulletin board, Topological entity modification, Event follow-up mechanisms, Generalized maps

\section{Introduction}

Over the last fifty years or so, geometric modeling systems have evolved significantly. Initially limited to 2D, they now include com-

\footnotetext{
*e-mail: babaali@sic.sp2mi.univ-poitiers.fr

†e-mail:marcheix@ensma.fr

‡e-mail:xavier.skapin@univ-poitiers.fr

$\S$ e-mail:yves.bertrand@univ-poitiers.fr
}

plex 3D functionalities, ranging from the simulation of physical phenomena to the recording and complete and automatic replay of parameterized processes of conception.

In the field of CAD (Computer Aided Design), some parametric systems have imposed themselves on the market, and the number of hours of development dedicated to these systems is so huge that it is very difficult to redevelop a new complete rival system.

Therefore, most new modeling systems which appear on the market basically use a ready -to-use geometric kernel (Parasolid, Acis, Cascade [Brunier-Coulin et al. 2000], etc.). These kernels supply the modeling system with an API (Application Programming Interface) containing a set of high level geometric operations (boolean operations, slot, chamfering, etc.).

Structurally, a geometric modeler is composed of two different levels of abstraction (see figure 1). The first level, in direct interaction with the user, includes the application layer which gathers the high level geometric operations. The second level, forming the core of the modeler, includes a geometric kernel based on a specific model (B-Rep, CSG, etc.).

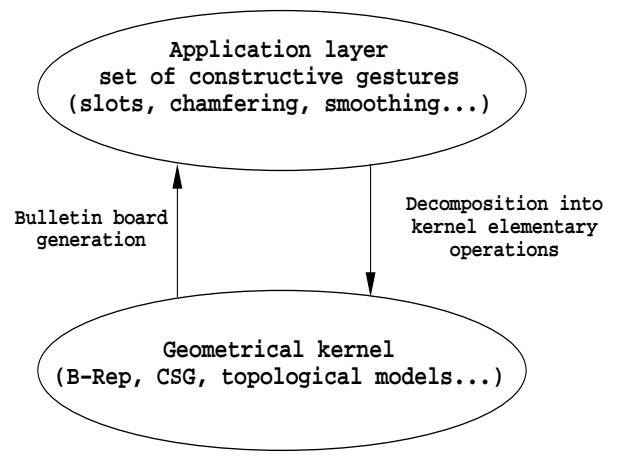

Figure 1: Geometric modeler structure.

Because of their complexity, the application layer's operations can generate important modifications on topological cells (vertices, edges, faces, volumes, etc.) of the objects. At the same time, many of these operations need to know precisely what has occurred to each topological cell belonging to objects given or resulting from a previous high level geometric operation. Next, at the end of each operation, the geometric kernel must provide a bulletin board describing cells' evolution through a list of events (split, merge, creation, deletion). For example, in the field of CAD, most parametric systems have developed home-grown solutions to resolve the persistent naming problems [Marcheix and Pierra 2002] (in our work, the persistent naming is just a case study). These solutions frequently use a graph in order to save face history during the conception process ([Kripac 1995], [Marcheix and Pierra 2002]). The construction of this graph needs to know how the faces of the geometric model evolve during an operation. In figure 2, block face $f_{1.1}$ is split into two faces $\left(f_{2.1}\right.$ and $\left.f_{2.2}\right)$ after the application of 
the difference boolean operation. The graph in figure $2 \mathrm{~b}$ stores this event that must be returned by the geometric kernel.

Currently, most geometric systems are developing new functionalities about standard construction procedures such as boolean operations for CSG or Euler operators for B-Rep modeling. These systems offer some solutions to the new needs expressed in many fields, such as CAD, architecture or geology. However, these solutions aren't satisfactory. Indeed, all commercial geometric kernels provide methods enabling the developer of a CAD system to retrieve a number of events occurred on cells. These kernels have their own scheme for detecting events, based on their own taxonomy of situations, heuristics and evolution rules. Little is known of their details, which are proprietary information, let alone of the underlying theory, if any. Generally, for example, the detected events are not generic for all cells' dimensions. This lack of underlying theory limits the possibility to extend the use of these kernels to new domains of investigation which need to detect events appearing on all $i$-cells (a cell of dimension $i$ where $i=0 . . n$ ) and different aggregates of connected i-cells. An interesting formalization work has been proposed in 2000 in the DJINN project [Armstrong et al. 2000]. This report proposes a complete specification of the different functions that must be provide by the interface of a geometric kernel. In this report, we can find the specification of several functions dedicated to the management of events occured on cells. This necessary formalization work emphases the necessity to provide these functions but it gives no answer on the way of generating in a correct an generic way these events in the geometric kernel. An underlying theory is necessary in order to guarantee the reliability and the completeness of the generated events.

In this paper, we are interested in the different modifications that a high level geometric operation generates on a modeler, particularly in the case of a topology based kernel. In order to solve this problem, we propose a generic model that enables to insert the complete list of events (we must omit any event) having occurred on cells of any dimension, and that belong to any topological model, into a bulletin board. The genericity and the complete independence with the used geometric model are based on four elementary mechanisms (split_elem, merge_elem, crea_elem, del_elem) allowing the generation of the bulletin board, whatever the geometric operation.

This paper is structured as follows. In section 2, we present our solution for the generic generation of bulletin boards into the topological kernel. Section 3 describes an implementation of this solution using the geometric kernel of the modeler Moka (web site: http://www.sic.sp2mi.univ-poitiers.fr/moka) that is based on the topological model of generalized maps [Lienhardt 1994]. This process permits us to integrate and validate the event follow-up mechanisms. We conclude in Section 4.

\section{List of events generation}

In this paper, our objective is to generalize the event follow-up mechanisms in order to describe a formalism robust enough to be implemented on any type of topological model.

The events which have occurred on the cells can be represented in the bulletin board as a list (every current geometric modeling system - Parasolid, Cascade, and so forth - has such a structure). This list must be complete and generic in any cell dimension. A bulletin board allows the tracking of topological cells' evolution inside a geometric model, after the application of a high level geometric operation (see figure 2a). To do so, the bulletin board links two sets of cells (respectively named starting cells and ending cells) with an event. Each event represents an interpretation of the topological evolution which has occurred on the starting cells (see figure $2 \mathrm{c}$ ).

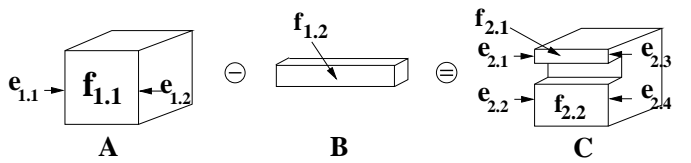

(a) Boolean operation of difference.

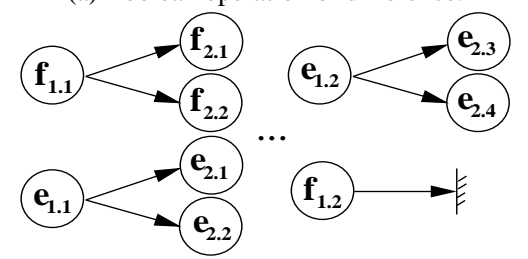

(b) Cell graph corresponding to a constructive gesture applied on the example of figure $2 \mathrm{a}$.

\begin{tabular}{|c|c|c|}
\hline Starting cells & Ending cells & Event \\
\hline$\left\{f_{1.1}\right\}$ & $\left\{f_{2.1}, f_{2.2}\right\}$ & face split \\
\hline$\left\{e_{1.1}\right\}$ & $\left\{e_{2.1}, e_{2.2}\right\}$ & edge split \\
\hline$\ldots$ & $\ldots$ & $\ldots$ \\
\hline$\left\{e_{1.2}\right\}$ & $\left\{e_{2.3}, e_{2.4}\right\}$ & edge split \\
\hline$\left\{f_{1.2}\right\}$ & \{\} & face deletion \\
\hline
\end{tabular}

(c) Bulletin board corresponding to a constructive gesture applied on the example of figure $2 \mathrm{a}$.

Figure 2: Events occured on cells after the application of a high level geometric operation.

In figure $2 \mathrm{a}$, the boolean operation between volumes $A$ and $B$ (high level geometric operation) generates several events and the corresponding bulletin board links several starting cells and ending cells sets (one pair set per line). Indeed, in the bulletin board shown in figure $2 \mathrm{c}$, the face split event gathers the starting set $\left\{f_{1.1}\right\}$ and the ending set $\left\{f_{2.1}, f_{2.2}\right\}$ (see line 1). Moreover, the face deletion event gathers the starting set $\left\{f_{1.2}\right\}$ and the empty ending set \{\} (see line 5).

\subsection{Prerequisites}

In a geometric modeler, we distinguish two levels of operations called "high level operation" and "elementary operation" (see figure 3). A high level operation (such as boolean operation) is a geometric procedure describing a complex geometric process. The topology resulting from this type of operation is highly dependent on geometry. Thus, the event list linking all starting cells and all ending cells can not be determined at this level (this is called "unpredictable"). For example, in figure $2 \mathrm{a}$, the split of face $f_{1.1}$ depends on the position of volume $B$. Thus, to find the events, after the application of the boolean operation, between all starting cells $\left\{f_{1.1}, e_{1.1}, \ldots, e_{1.2}, f_{1.2}\right\}$ and all ending cells $\left\{f_{2.1}, f_{2.2}, \ldots, e_{2.3}, f_{2.4}\right\}$ (see figure 2c) is unfeasable. Available in application layer (see figure 1), a high level operation must be processed at the geometric kernel of a modeler with a set of elementary operations.

Unlike a high level operation, an elementary operation generates a predictable and finite list of events (figure 4 shows an elementary operation of face splitting, applied on $F$ ). This list includes the events of creation, deletion, merge and split of cells. In figure 4 , we can only find two edge split $\left(A_{1}, A_{2}\right)$, one face split $(F)$ and two edge creation $\left(A_{3}, A_{4}\right)$ events. In order to transcribe the events on the bulletin board, we define four mechanisms for following up events. Each of them is associated with the elementary operation (see figure 3) which generates the corresponding event. Indeed, the events' split, merge, creation and deletion of cells respectively match the mechanisms split_elem, merge_elem, 


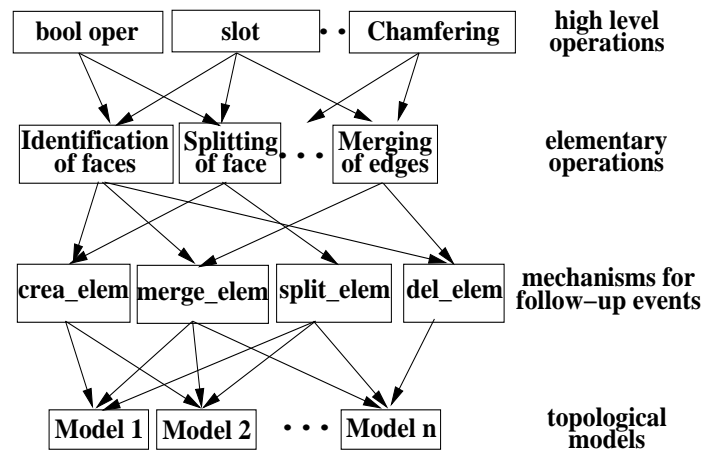

Figure 3: Architecture of our model to generate the list of events which have occurred on cells.

crea_elem and del_elem.

\subsection{Mechanisms for event follow-up}

To define these mechanisms, we need to introduce the definitions below:

- ID_Cell: an infinite set of identifiers which allow the characterization of every cell.

- ID_used: the set of the identifiers used during a high level operation (ID_used $\subset$ ID_Cell).

- Dim_Max: the maximal dimension of cells in the topological model. In $2 D$ space (resp. in $3 D$ space), Dim_Max $=2$ (resp. Dim_Max $=3$ ).

After the application of any high level operation $O$, a set of events have occurred on the cells of the topological model. The identification of these elements consists in finding the links between the starting cells and the ending cells of $O$. We proceed as follows:

1. characterize each cell $c$ by an identifier $i d\left(i d \in I D \_C e l l\right)$;

2. associate the pair (set_id,dim) with $i d$. set_id represents a set of identifiers refering to all the cells from which $c$ has been taken. set_id can be an empty set if $c$ has just been created and $\operatorname{dim}$ represents the dimension of $c$. In figure 2a, pair $\left(\left\{f_{1.1}\right\}, 2\right)$ must be associated with an identifier $f_{2.2}$ because face $f_{2.2}$ originated from face $f_{1.1}$.

The faces $F, F_{1}$ and $F_{2}$ in figure 4 a are respectively characterized by the identifiers $i d_{1}, i d_{4}$ and $i d_{5}$ (see figure $4 \mathrm{~b}$ ). Identifier $i d_{1}$ is associated with the pair $\left(\left\{i d_{1}\right\}, 2\right)$ because we apply the initialization process of ancestors described below. Faces $F_{1}$ and $F_{2}$ derive from $F$, so both pairs $\left(\left\{i d_{1}\right\}, 2\right)$ and $\left(\left\{i d_{1}\right\}, 2\right)$ are respectively associated with identifiers $i d_{4}$ and $i d_{5}$.

The relations called ancestor and dimension are respectively defined by:

$$
\begin{gathered}
\text { ancestor }:\left\{\begin{array}{c}
I D \_C e l l \rightarrow \mathfrak{P}(\text { ID_Cell }) \\
i d \mapsto \text { ancestor }(\text { id })=\text { set_id }
\end{array}\right. \text { and } \\
\text { dimension }:\left\{\begin{array}{c}
I D \_C e l l \rightarrow[0, \text { Dim_Max }] \\
i d \mapsto \text { dimension }(\text { id })=\text { dim }
\end{array}\right.
\end{gathered}
$$$$
\left(\mathfrak{P}\left(I D \_C e l l\right)\right. \text { symbolizes the power set of ID_Cell). }
$$

The ancestor of $c$ must be initialized at the beginning of a high level operation. This process consists in giving the value of singleton $\{i d\}$ to each set set_id. We formalize this initialization by: $\forall i d \in I D \_C e l l ; i d \in D_{\text {ancestor }} \Rightarrow$ ancestor $(i d)=\{i d\}$ ( $D_{\text {ancestor }}$ is the range of function ancestor).
Let us consider the example shown in figure 4 and let us assume that we apply the initialization process on faces $F_{1}$ and $F_{2}$ at the beginning of the following high level operation. Identifier $i d_{4}$ (resp. $i d_{5}$ ) is associated with the set $\left\{i d_{4}\right\}$ (resp. $\left\{i d_{5}\right\}$ ).

All these definitions allow us to define mechanisms of an event follow-up formally. We only describe the mechanism "split_elem" because the principle is the same for every other mechanism.

\subsubsection{Mechanism "split_elem"}

Splitting cell $c$ of dimension $\operatorname{dim}(\operatorname{dim} \neq 0)$ results in two new cells $c_{1}$ and $c_{2}$ with the same dimension. Identifier $i d$ (resp. identifiers $i d_{1}$ and $i d_{2}$ ) which characterizes the cell $c$ (resp. cells $c_{1}$ and $c_{2}$ ) is associated with the set of identifiers set_id (resp. two sets of identifiers set_id $d_{1}$ and set_id $d_{2}$ ). The mechanism split_elem allows to define $s e t_{-} i d_{1}$ and $s_{-} t_{-} i d_{2}$ such as set_id $_{1} \subseteq$ set_id,

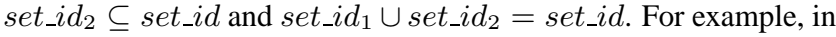
figure 4 , we assume that we initialize the ancestor of cell $F$ before starting the face splitting elementary operation.
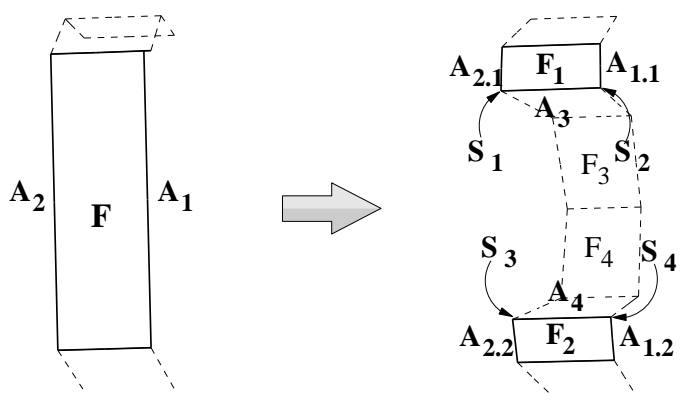

(a)

\begin{tabular}{|c|c|c|c|}
\hline Cell & $\begin{array}{c}\text { Identifier } \\
i d_{i}\end{array}$ & $\begin{array}{c}\text { Set of identifiers } \\
\left(\text { set_id }_{i}\right)\end{array}$ & Dimension \\
\hline$F$ & $i d_{1}$ & $\left\{i d_{1}\right\}$ & 2 \\
\hline$A_{1}$ & $i d_{2}$ & $\left\{i d_{2}\right\}$ & 1 \\
\hline$A_{2}$ & $i d_{3}$ & $\left\{i d_{3}\right\}$ & 1 \\
\hline$F_{1}$ & $i d_{4}$ & $\left\{i d_{1}\right\}$ & 2 \\
\hline$F_{2}$ & $i d_{5}$ & $\left\{i d_{1}\right\}$ & 2 \\
\hline$A_{3}$ & $i d_{6}$ & \{\} & 1 \\
\hline$A_{4}$ & $i d_{7}$ & \{\} & 1 \\
\hline$A_{1.1}$ & $i d_{8}$ & $\left\{i d_{2}\right\}$ & 1 \\
\hline$A_{1.2}$ & $i d_{9}$ & $\left\{i d_{2}\right\}$ & 1 \\
\hline$A_{2.1}$ & $i d_{10}$ & $\left\{i d_{3}\right\}$ & 1 \\
\hline$A_{2.2}$ & $i d_{11}$ & $\left\{i d_{3}\right\}$ & 1 \\
\hline$S_{1}$ & $i d_{12}$ & \{\} & 0 \\
\hline$S_{2}$ & $i d_{13}$ & \{\} & 0 \\
\hline$S_{3}$ & $i d_{14}$ & \{\} & 0 \\
\hline$S_{4}$ & $i d_{15}$ & \{\} & 0 \\
\hline
\end{tabular}

(b)

Figure 4: Elementary operation "face splitting”. a) Boundary representation of split face $F . b)$ Information associated with some cells of the model shown in (a).

set_id $d_{1}$ is initialized with the singleton $\left\{i d_{1}\right\}$ (id $d_{1}$ characterizing $F)$. After splitting $F$, identifiers $i d_{4}$ and $i d_{5}$, which respectively characterize faces $F_{1}$ and $F_{2}$, are associated with the same set set_id ${ }_{1}\left(\right.$ set_id $_{4}=$ set_id $_{5}=$ set_$\left._{-} i d_{1}\right)$. Both $F_{1}$ and $F_{2}$ have $F$ as ancestor and the sets of identifiers associated with $i d_{4}$ and $i d_{5}$ follow the preconditions $\left(\left\{i d_{1}\right\} \subseteq\left\{i d_{1}\right\}\right.$ and $\left.\left\{i d_{1}\right\} \cup\left\{i d_{1}\right\}=\left\{i d_{1}\right\}\right)$.

The mechanism "split_elem", described by algorithm 1, adds new relations linking the identifiers which characterize cells $c_{1}$ and $c_{2}$ 


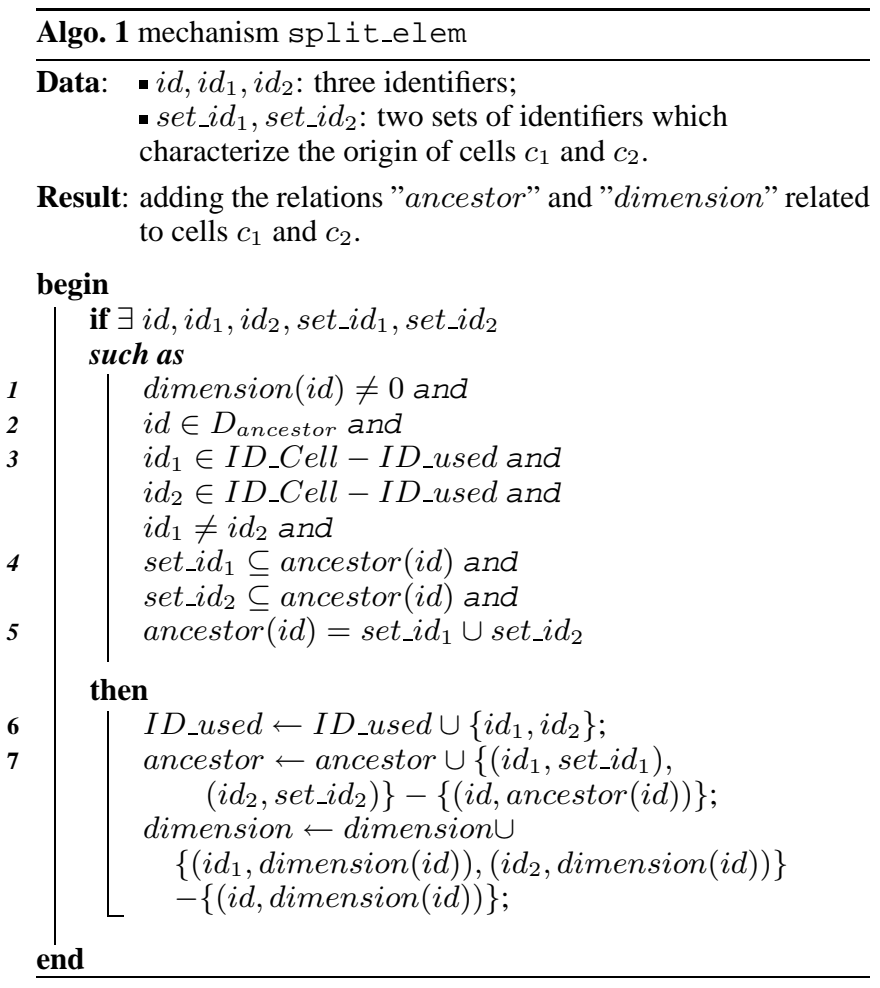

with their origin and their dimension (in order to update the functions ancestor and dimension defined in section 2.2). This mechanism takes the identifier $i d$ (resp. $i d_{1}$ and $i d_{2}$ ) characterizing cell $c$ (resp. $c_{1}$ and $c_{2}$ ) and the sets set_id $d_{1}$ and set_id $d_{2}$, respectively characterizing the origin of cells $c_{1}$ and $c_{2}$, as parameters. Points 1 to 5 present the preconditions of algorithm 1; the algorithm itself is described in points 6 and 7 .

1. We do not consider the mechanism split_elem on 0dimensional cells. Indeed, the split of a vertex has, from a semantic point of view, no meaning.

2. id belongs to the range of function ancestor.

3. $i d_{1}$ and $i d_{2}$ are two new identifiers not yet used.

4. The set set_id $_{1}$ (resp. set_id ${ }_{2}$ ) characterizes the ancestor of $c_{1}$ (resp. $c_{2}$ ) and is included in the set which characterizes the ancestor of $c$.

5. The union of set_id 1 and set_id $d_{2}$ corresponds to the set ancestor $(i d)$ which gathers the ancestors of $c$. Therefore, all the ancestors of $c$ are added to the ancestors of both $c_{1}$ and $c_{2}$. Only the implementation of the mechanism, in accordance with a specific topological model, makes it possible to determine the exact contents of sets set_id $d_{1}$ and set_id $d_{2}$.

6. $i d_{1}$ and $i d_{2}$ are added to set ID_used.

7. The values of ancestor and dimension are updated by adding (resp. subtracting) the ancestor and the dimension of both $c_{1}$ and $c_{2}$ (resp. $c$ ).

\subsection{Bulletin board generation}

After relating each identifier $i d$ (and thus each cell $c$ ) of the geometric model to a set of identifiers set_id (resp. a dimension dim) characterizing the ancestor (resp. dimension) of $c$, we check every cell of this model at the end of high level operations (i.e. a posteriori), in order to retrieve every relation binding all identifiers $i d$ to their ancestor set of identifiers set_id and to the dimension $\operatorname{dim}$ of the cell they reference. These relations must be inserted in the bulletin board, in order to distinguish the events occurring both on the starting cells and the ending cells during any high level operation.

Of course, when deleting a cell, the recovery of the relations described just before cannot be only carried out a posteriori. Indeed, deleting a cell implies the loss of information which were associated with this cell. To solve this issue, first we propose to check the cells at the beginning of high level operations. The sets characterizing the ancestor of each identifier are gathered into a set $D_{1}$. Therefore, at the end of the high level operation, we gather the identifiers characterizing the ancestors of every existing cell in a second set $D_{2}$ and thus, we can determine which cells have been deleted by defining the set $D\left(D=D_{1}-D_{2}\right)$.

With the formalism defined in the previous section, checking cells can be considered as a checking set $E$ defined by $E=$ $\left\{(i d\right.$, ancestor $\left.(i d)) \mid i d \in D_{\text {ancestor }}\right\}$. Some elements of $E$ are gathered in $n$ subsets ( $n$ may be equal to 0 ). Each subset, called $S_{E_{k}}(k \in[0, n])$, contains elements $x$ of $E(x=(i d$, set_id $))$ which have the same set_id. Therefore, all cells with the same ancestor are gathered. Formally, $S_{E_{k}}$ are defined as:

$S_{E_{k}}=\left\{\forall x_{i}, x_{j} \mid\left(x_{i}, x_{j}\right) \in S_{E_{k}}^{2} \Rightarrow\left(x_{i}, x_{j}\right) \in E^{2}\right.$ and $x_{i}=$ $\left(i d_{i}\right.$, set_id $\left._{i}\right)$ and $x_{j}=\left(i d_{j}\right.$, set $\left._{-} i d_{j}\right)$ and set_id $d_{i}=$ set $_{-} i d_{j}$ and set_idi $\neq \emptyset$.

Figure $4 \mathrm{~b}$ shows the information associated with the cells used by the elementary operation of face splitting. Set $E$, built at the end of the high level operation, contains pairs $\left(i d_{4},\left\{i d_{1}\right\}\right)$, $\left(i d_{5},\left\{i d_{1}\right\}\right),\left(i d_{6},\{\}\right),\left(i d_{7},\{\}\right),\left(i d_{8},\left\{i d_{2}\right\}\right),\left(i d_{9},\left\{i d_{2}\right\}\right)$, $\left(i d_{10},\left\{i d_{3}\right\}\right),\left(i d_{11},\left\{i d_{3}\right\}\right), \ldots,\left(i d_{15},\{\}\right)$. With these pairs, we can define subsets $S_{E_{0}}=\left\{\left(i d_{4},\left\{i d_{1}\right\}\right),\left(i d_{5},\left\{i d_{1}\right\}\right)\right\}$, $S_{E_{1}}=\left\{\left(i d_{8},\left\{i d_{2}\right\}\right),\left(i d_{9},\left\{i d_{2}\right\}\right)\right\}$ and $S_{E_{2}}=\left\{\left(i d_{10},\left\{i d_{3}\right\}\right)\right.$, $\left.\left(i d_{11},\left\{i d_{3}\right\}\right)\right\}$. Every element of each subset has the same set_id.

We build a new set called $S$ from subsets $S_{E_{k}}$. Each pair $\left(A_{k}, B_{k}\right)$ of $S$ gathers elements contained in the previously defined subset $S_{E_{k}}\left(A_{k}\right.$ and $B_{k}$ are two sets of identifiers defined from $S_{E_{k}}$ ). Therefore, $S$ is defined: $S=\left\{x_{k}=\left(A_{k}, B_{k}\right) \mid k \in[0, n]\right.$ and $\forall x=\left(\right.$ id, set_id) $\mid x \in S_{E_{k}} \Rightarrow i d \in B_{k}$ and $A_{k}=$ set_id\}. This set gathers all events of cell split. In figure 4, $S$ (built from the subsets $S_{E_{0}}, S_{E_{1}}$ and $S_{E_{2}}$ ) contains elements $\left(A_{0}, B_{0}\right)=\left(\left\{i d_{1}\right\},\left\{i d_{4}, i d_{5}\right\}\right),\left(A_{1}, B_{1}\right)=\left(\left\{i d_{2}\right\},\left\{i d_{8}, i d_{9}\right\}\right)$ and $\left(A_{2}, B_{2}\right)=\left(\left\{i d_{3}\right\},\left\{i d_{10}, i d_{11}\right\}\right)$. From set $D$ of deleted cells, we also build that set $Z$ defined by: $Z=\{\forall x \mid x \in D \Rightarrow$ $(\{x\},\{\}) \in Z\}$.

For every other case (create and merge events), we define set $A$ by: $A=\left\{\forall x \mid x \in E-\bigcup_{k=0}^{n} S_{E_{k}} \Rightarrow x=(i d\right.$, set_id) and (set_id, $\{i d\}) \in A\}$. In figure 4 , set $E-\bigcup_{k=0}^{n} S_{E_{k}}$ contains elements $\left(i d_{6},\{\}\right),\left(i d_{7},\{\}\right),\left(i d_{12},\{\}\right),\left(i d_{13},\{\}\right),\left(i d_{14},\{\}\right)$ and $\left(i d_{15},\{\}\right)$ (see figure $\left.4 \mathrm{~b}\right)$. Therefore, $A$ contains $\left(\{\},\left\{i d_{6}\right\}\right)$, $\left(\{\},\left\{i d_{7}\right\}\right),\left(\{\},\left\{i d_{12}\right\}\right),\left(\{\},\left\{i d_{13}\right\}\right),\left(\{\},\left\{i d_{14}\right\}\right)$ and $(\{\}$, $\left.\left\{i d_{15}\right\}\right)$.

Eventually, every line of the bulletin board matches an element of set $B B$ defined by: $B B=S \cup Z \cup A$. Indeed, for each pair $x=$ $\left(e_{1}, e_{2}\right)$ of $B B$, we put $e_{1}$ among the starting cells and $e_{2}$ among the ending cells of the bulletin board. In figure 4 , the elements of $S$ are located on the first line of table 1, the elements of set $A$ are located on the last line and $Z$ does not contain any element. 


\begin{tabular}{|c|c|c|}
\hline Starting cells & Ending cells & Remark \\
\hline$\left\{i d_{1}\right\}$ & $\left\{i d_{4}, i d_{5}\right\}$ & $\left(\left\{i d_{1}\right\},\left\{i d_{4}, i d_{5}\right\}\right) \in S$ \\
\hline$\ldots$ & $\ldots$ & $\ldots$ \\
\hline\{\} & $\left\{i d_{12}\right\}$ & $\left(\{\},\left\{i d_{12}\right\}\right) \in A$ \\
\hline
\end{tabular}

Table 1: Bulletin board generated after applying a high level operation which contains the elementary operation shown in figure 4.

\subsubsection{Interpretation of events}

After inserting the elements of set $B B$ into the bulletin board, the next step is to identify which events have occured (creation, deletion, split, merge, change) by looking at the cells located on the same line in the bulletin board. Our criterion used for the identification of events is the cardinality of both the set of starting cells (card $(S C)$ ) and the set of ending cells (card $(E C)$ ) inside the bulletin board. The identification process for each event is described like this:

- if $S C$ is an empty set then the corresponding event is creation (see line 3 in Tab. 1).

- if $E C$ is an empty set then the corresponding event is deletion.

- if $\operatorname{card}(S C)=1$ and $\operatorname{card}(E C)>1$ then the corresponding event is split (see line 1 in Tab. 1).

- if $\operatorname{card}(E C)=1$ and $\operatorname{card}(S C)>1$ then the corresponding event is merge.

- if $\operatorname{card}(E C)=1$ and $\operatorname{card}(S C)=1$ then the corresponding event is change.

Therefore, we deduce that: (1) set $S$ contains only pairs describing the event "cell split" (see Tab. 1); (2) set $Z$ exclusively contains pairs describing the event "cell deletion"; (3) set $A$ contains pairs describing events of "cells creation" and "cell merge".

In the following section, we describe an implementation of the model presented above. We use the kernel of the modeler Moka (based on generalized maps) in order to show a case study.

\section{Case study: generalized maps}

The choice of generalized maps (G-maps) model as a study case is not fortuitous. Indeed, this model (see figure 5) enables to subdivide an $\mathrm{nD}$ space (in the example below, we limit us to the $3 \mathrm{D}$ case) into n-dimensional quasi-manifolds, orientable or not, with or without boundary [Lienhardt 1994]. Therefore, we have chosen this model for its generality, although we could have used any other B-rep model. In order to implement the previously described solution on the G-maps model, we have defined a structure called tag. In this section, we describe this structure as well as the various follow-up event mechanisms.

\subsection{Generalized maps model}

An $n$-dimensional generalized map is a set of abstract elements, called darts, and applications defined on these darts:

Definition 1 (Generalized map) Let $n \geq 0$. A n-dimensional generalized map (or $n$-G-map) is $G=\left(B, \alpha_{0}, \ldots, \alpha_{n}\right)$ where:

1. $B$ is a finite set of darts;

2. $\forall i, 0 \leq i \leq n, \alpha_{i}$ is an involution ${ }^{1}$ on $B$;

\footnotetext{
${ }^{1}$ An involution $f$ on $S$ is a one mapping from $S$ onto $S$ such that $f=$
}
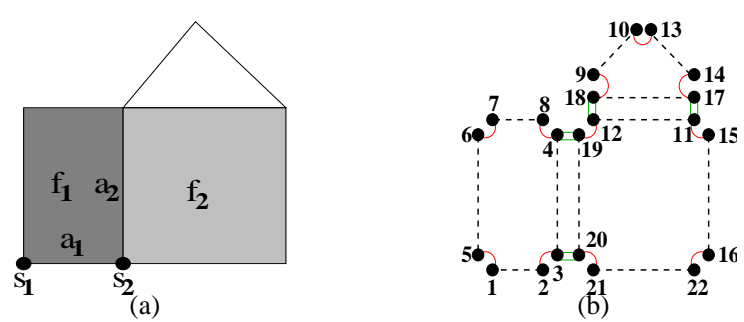

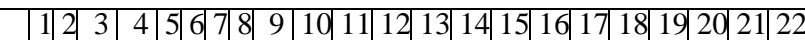
\begin{tabular}{llllllllllllllllllllllllll}
\hline$\alpha_{0}$ & 2 & 1 & 4 & 3 & 6 & 5 & 8 & 7 & 10 & 9 & 12 & 11 & 14 & 13 & 16 & 15 & 18 & 17 & 20 & 19 & 22 & 21
\end{tabular}

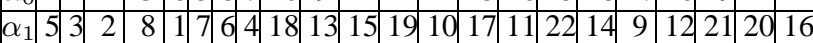

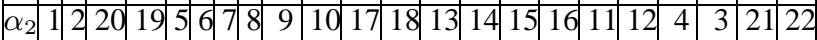

Figure 5: (a) A 2D subdivision. (b) The corresponding 2-G-map (involutions are given explicitly in the array). Darts are represented by dots. Two darts in relation by $\alpha_{0}$ share a dashed line (ex. darts 1 and 2). Two darts in relation by $\alpha_{1}$ share a red arc (ex. darts 2 and 3). Two distinct darts in relation by $\alpha_{2}$ share a pair of green lines (ex. darts 3 and 20); otherwise, the dart is its own image by $\alpha_{2}$ (ex. dart 2). Dart 1 corresponds to $\left(s_{1}, a_{1}, f_{1}\right)$, dart $2=1 \alpha_{0}$ corresponds to $\left(s_{2}, a_{1}, f_{1}\right), 3=2 \alpha_{1}$ corresponds to $\left(s_{2}, a_{2}, f_{1}\right)$, and $20=3 \alpha_{2}$ corresponds to $\left(s_{2}, a_{2}, f_{2}\right)$. The vertex incident to dart 2 is $\left\langle\alpha_{1}, \alpha_{2}\right\rangle(2)=\{2,3,20,21\}$, the edge incident to dart 3 is $\left\langle\alpha_{0}, \alpha_{2}\right\rangle(3)=\{3,4,19,20\}$, and the face incident to dart 9 is $\left\langle\alpha_{0}, \alpha_{1}\right\rangle(9)=\{9,10,13,14,17,18\}$.

\section{3. $\forall i, j, 0 \leq i<i+2 \leq j \leq n, \alpha_{i} \alpha_{j}$ is an involution.}

Let $G$ be an n-G-map, and $S$ be the corresponding subdivison. Intuitively, a dart of $G$ corresponds to an $(n+1)$-tuple of cells $\left(c_{0}, \ldots, c_{n}\right)$, where $c_{i}$ is an $i$-dimensional cell that belongs to the boundary of $c_{i+1}$ [Brisson 1993] (Fig. 5). $\alpha_{1}$ associates darts corresponding with $\left(c_{0}, \ldots, c_{n}\right)$ and $\left(c_{0}^{\prime}, \ldots, c_{n}^{\prime}\right)$, where $c_{j}=c_{j}^{\prime}$ for $j \neq i$, and $c_{i} \neq c_{i}^{\prime}\left(\alpha_{i}\right.$ swaps the two $i$-cells that are incident to the same $(i-1)$ and $(i+1)$-cells). When two darts $b_{1}$ and $b_{2}$ are such that $b_{1} \alpha_{i}=b_{2}(0 \leq i \leq n), b_{1}$ is said $i$-sewn with $b_{2}$. G-maps represent cells in an implicit way:

Definition 2 (i-cell) Let $G$ be an $n$-G-map, $b$ a dart and $i \in N=$ $\{0, ., n\}$. The $i$-cell incident to $b$ is the orbit ${ }^{2}$

$$
\langle\rangle_{N-\{i\}}(b)=\left\langle\alpha_{0}, \ldots, \alpha_{i-1}, \alpha_{i+1}, \ldots, \alpha_{n}\right\rangle(b)
$$

Intuitively, an $i$-cell is the set of all darts which can be reached starting from b, by using any combination of all involutions except $\alpha_{i}$. The set of $i$-cells is a partition of the darts of the G-map, for each $i$ between 0 and n. Two cells are disjoined if their intersection is empty, i.e. when no dart is shared by the cells. More precisions about G-maps are provided in Ref. [Lienhardt 1994].

\subsection{Solution brought by the "tag" structure}

Tracking the modifications of topological $i$-cells [Lienhardt 1994] $(0 \leq i \leq 3)$ of a G-map $G$ can be done by means of tracking the modifications on the darts constituting these $i$-cells. It consists in calculating which cells contain this dart before and after a high level operation. To do so, a data structure called "tag" is defined. It is a pair of quadruplets of integers related to each dart $b \in G$. The

\footnotetext{
${ }^{2}$ Let $\left\{\Pi_{0}, \ldots, \Pi_{n}\right\}$ be a set of permutations on $B$. The orbit of an element $b$ relatively to this set of permutations is $\left\langle\Pi_{0}, \ldots, \Pi_{n}\right\rangle(b)=$ $\left\{\Phi(b), \Phi \in\left\langle\Pi_{0}, \ldots, \Pi_{n}\right\rangle\right\}$, where $\left\langle\Pi_{0}, \ldots, \Pi_{n}\right\rangle$ denotes the group of permutations generated by $\left\{\Pi_{0}, \ldots, \Pi_{n}\right\}$.
} 
two components of this pair are respectively called "current_tag" and "ancestor_tag", and are defined as:

1. current_tag: it corresponds to quadruplet (cur_id $d_{0}, c u r_{-} i d_{1}$, cur_id $d_{2}$, cur_id $\left.d_{3}\right)$ and respectively characterizes each $i$-cell $c_{i}(0 \leq i \leq 3)$ containing dart $b$ by an identifier cur_i $i d_{i}$;

2. ancestor_tag: it corresponds to quadruplet (anc_id $d_{0}, a n c_{-} i d_{1}$, $\left.a n c_{-} i d_{2}, a n c_{\_} i d_{3}\right)$ and respectively characterizes each $i$-cell, ancestor of $c_{i}$, by an identifier $a n c_{-} i d_{i}$ ( $i$ matches a dimension of $\left.c_{i}\right)$.

Figure 6 shows the tags related to the darts constituting an edge before and after an elementary operation of edge splitting. For example, quadruplet $(4,1,2,3)$ corresponding to the current_tag of dart $b_{1}$ in figure $6 \mathrm{a}$, means that $b_{1}$ belongs to the 0 -cell number 4 , to the 1 -cell number 1 , to the 2 -cell number 2 and to the 3 -cell number 3 . This principle is similar for every other dart.

All the current_tags related to darts belonging to an $i$-cell $c_{i}$ have the same identifier cur_id $d_{i}$. In figure 6 a (resp. figure $6 \mathrm{c}$ ), darts $b_{1}$ and $b_{2}$ (resp. darts $b_{3}$ and $b_{4}$ ) forming 1-cell $A$ (resp. 0-cell $S$ ) have the same identifier $c u r \_i d_{1} 1$ (resp. the same identifier $c u r_{-} i d_{0} 8$ ). However, an $i$-cell gathers a set of identifiers $a n c_{-} i d_{i}$. Indeed, $c_{i}$ contains ancestor_tags that do not necessarily have the same identifier $a n c_{-} i d_{i}$. Moreover, this set can be empty when all identifiers $a n c \_i d_{i}$ are set to value ID_NULL (ID_NULL is a particular value which means that an identifier has no value). In figure $6 \mathrm{c}$, darts $b_{3}$ and $b_{4}$ have ancestor_tags that contain an $a n c_{-} i d_{0}$ set to ID_NULL (represented by the character "-"). The current and ancestor tag structures allow one to associate an identifier $c u r \_i d_{i}$ (which characterizes each $i$-cell $c_{i}$ ) with a set of identifiers set_id characterizing the ancestor of $c_{i}$.

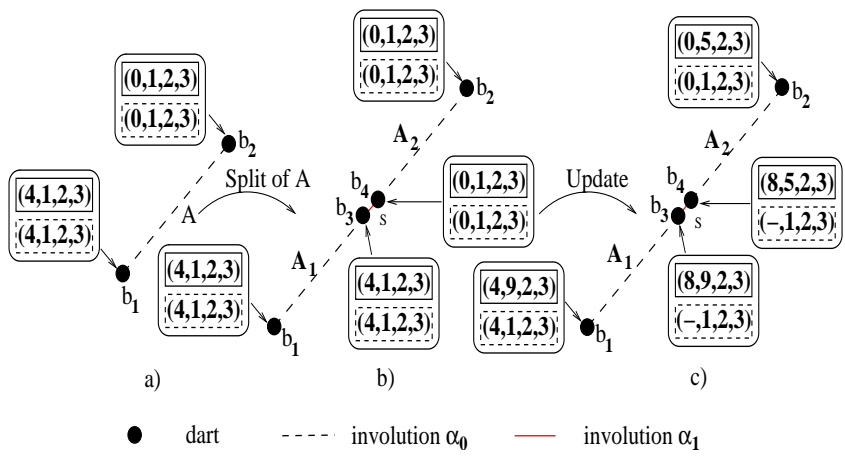

Figure 6: Edge splitting. For every dart, the current_tags are surrounded with full border, while the ancestor_tags are surrounded with dashed border. a) Darts $b_{1}$ and $b_{2}$ form edge $A$ before the split. b) $A$ is split in $A_{1}=\left(b_{1}, b_{3}=\alpha_{0}\left(b_{1}\right)\right)$ and $A_{2}=\left(b_{2}, b_{4}=\right.$ $\left.\alpha_{0}\left(b_{2}\right)\right)$. Vertex $S$ connecting $A_{1}$ and $A_{2}$ is made of both $b_{3}$ and $b_{4}=\alpha_{1}\left(b_{3}\right) . b_{1}$ and $b_{2}$ save and propagate their tags to the new darts of $A_{1}$ and $A_{2}: b_{3}$ and $b_{4}$. c) Update of current_tag of $b_{1}$ and $b_{2}$, and update of both tags associated with $b_{3}$ and $b_{4}$.

For each dimension, the identifiers of cells are defined modulo 4 because we work in a $3 \mathrm{D}$ space. Thus, we can directly deduce the dimension of the cell from its identifier. More precisely, each $i$ cell has got an identifier cur $_{-} i d_{i}$ such that cur $_{-} i d_{i} \bmod 4=i$. In that way, we have implemented the function dimension defined in section 2.2. Then, we can update the current_tag structure on the darts of figure $6 \mathrm{c}$ : edge $A_{1}$, created during the split, is characterized by the number $9(9 \bmod 4=1)$, and this value is set to identifier cur_id $d_{1}$ of $b_{1}$ and $b_{3}$. This principle is the same for edge $A_{2}$ : it receives the number 5 , and this value is set to identifier cur $_{-} i d_{1}$ of $b_{2}$ and $b_{4}$. Eventually, vertex $S$ receives the number 8 and this value is set to identifier $c u r_{-} i d_{0}$ of $b_{3}$ and $b_{4}$.

The next section is dedicated to the implementation of the elementary mechanisms for following up events to Moka during the elementary operations forming the co-refinement high level operation.

\subsection{Co-refinement high level operation}

Until now, in order to realise a machining operations on an geometric objects, we use classic boolean operations such as the union, the difference or the intersection. The co-refinement is a common denominator for the calculation of an intersection and allows to obtain, after an extraction (this step allows to save only one volume resulting from a $3 \mathrm{D}$ subdivision of the co-refinement operation) phase, the same results as the three preceding operations.

The co-refinement operation in dimension 3 consists in generating the spatial subdivision resulting from the intersection between two original 3D subdivisions. Generally, applying co-refinement consists in computing the intersections of faces of the original subdivisions and updating the topology to obtain a valid final subdivision.

Most existing works in this field ([Perrin 2005], [Gardan et al. 2003], [Kitajima and Yamaguchi 1992], [Mantyla and Tamminen 1983], [Ma and Tang 1988]) are essentialy based on applying the boolean operation on two volumes. They let us classify the different parts of an object against the interior or the exterior of another object. In most cases, these methods only allow to process surfacic objects (ie. 2D topological objects having 3D embedding) and to build a single volume from the initial objects.

As in the case of boolean operations, many problems can occur when computing intersections. Indeed, the 3D co-refinement algorithm we use is based on a method frequently used in the algorithms associated with boolean operations on surfacic objects, but Guiard [Guiard 2006] has extended this method for 3D grids, with a new algorithm based on the intersections of pairs of faces, and has implemented it with the G-maps model. The use of boolean operations is fundamental in many modelers. Moreover, the important number and the unpredictable behaviour of events which occur during the co-refinement operation makes them difficult, and thus particularly interesting, to track.

The operation of co-refinement is decomposed in the following way: insertion of a dangling edge into a face, edge splitting, face splitting and face identification. This set represents only a part of the elementary operations that can be defined in a geometric kernel and particularly in the case of a G-map kernel. However, in order to integrate the mechanisms of events' follow-up, the approach described in this paper is still the same and can easily be extended to the whole set of elementary operations. We present here the necessary functions to describe every elementary operation used in the co-refinement algorithm:

- Copying_Tag ( $b$ : Dart, $O$ : Orbit): this function propagates current_tag and ancestor_tag related to dart $b$ to all darts constituting orbit $O$.

- Rep_Anc_ID (O : Orbit, $d$ : [0..Dim_max], id : ID_Cell) : this function updates the identifier $a n c_{-} i d_{d}$ of each ancestor_tag related to the darts constituting orbit $O$ with value $i d$ ( $i d$ must pass the condition: $i d \bmod 4=d$ ).

- Rep_Cur_ID ( $O$ : Orbit, $d$ : [O..Dim_max], id : ID_Cell) : This function has the same role as function Rep_Anc_ID. However, it uses the current_tag structure.

We only describe the elementary operation "face splitting" because the principle is similar for every other elementary operation. 


\subsubsection{Face splitting}

Splitting a face $F$ results in the insertion of an edge $A$ into $F$. The result of this split is the generation of two new faces incident to $F$ and called $F_{1}$ and $F_{2}$ (see figure 7). Mechanisms split_elem and crea_elem relate to this function.

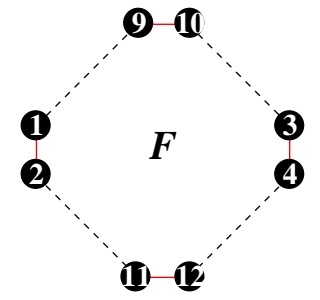

(a)

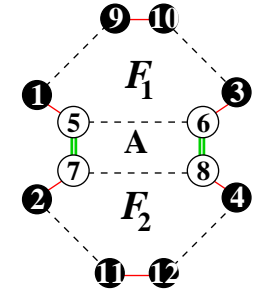

(b)
Before

\begin{tabular}{|c|c|c|c|c|c|c|c|c|}
\hline dart & \multicolumn{3}{|c|}{ ancestor_tag } & \multicolumn{4}{c|}{ current_tag } \\
\hline 1 & 4 & 5 & 2 & 3 & 4 & 5 & 2 & 3 \\
\hline 2 & 4 & 9 & 2 & 3 & 4 & 9 & 2 & 3 \\
\hline 3 & 8 & 13 & 2 & 3 & 8 & 13 & 2 & 3 \\
\hline 4 & 8 & 17 & 2 & 3 & 8 & 17 & 2 & 3 \\
\hline 9 & 12 & 5 & 2 & 3 & 12 & 5 & 2 & 3 \\
\hline 10 & 12 & 13 & 2 & 3 & 12 & 13 & 2 & 3 \\
\hline 11 & 16 & 9 & 2 & 3 & 16 & 9 & 2 & 3 \\
\hline 12 & 16 & 17 & 2 & 3 & 16 & 17 & 2 & 3 \\
\hline
\end{tabular}

\begin{tabular}{|c|c|c|c|c|c|c|c|c|}
\hline dart & \multicolumn{4}{|c|}{ ancestor_tag } & \multicolumn{4}{c|}{ current_tag } \\
\hline 1 & 4 & 5 & 2 & 3 & 4 & 5 & 6 & 3 \\
\hline 2 & 4 & 9 & 2 & 3 & 4 & 9 & 10 & 3 \\
\hline 3 & 8 & 13 & 2 & 3 & 8 & 13 & 6 & 3 \\
\hline 4 & 8 & 17 & 2 & 3 & 8 & 17 & 10 & 3 \\
\hline 5 & 4 & - & 2 & 3 & 4 & 21 & 6 & 3 \\
\hline 6 & 8 & - & 2 & 3 & 8 & 21 & 6 & 3 \\
\hline 7 & 4 & - & 2 & 3 & 4 & 21 & 10 & 3 \\
\hline 8 & 8 & - & 2 & 3 & 8 & 21 & 10 & 3 \\
\hline 9 & 12 & 5 & 2 & 3 & 12 & 5 & 6 & 3 \\
\hline 10 & 12 & 13 & 2 & 3 & 12 & 13 & 6 & 3 \\
\hline 12 & 16 & 17 & 2 & 3 & 16 & 17 & 10 & 3 \\
\hline
\end{tabular}

(c)

Figure 7: Face splitting. (a) Darts $\{1,2,3,4,9,10,11,12\}$ belong to $F$ before the split. (b) $F$ is split into $F_{1}$ (composed of darts $\{1,3,5,6,9,10\})$ and $F_{2}$ (composed of darts $\{2,4,7,8,11,12\}$ ). Edge A, incident to $F_{1}$ and $F_{2}$, is made of darts $\{5,6,7,8\}$. (c) The content of each tag related to one of these darts at before and after face splitting.

This elementary operation is performed by:

SplitFace $\left(b_{1}, b_{2}:\right.$ Dart $) \rightarrow$ Dart: this function splits the face incident to darts $b_{1}$ and $b_{2}$. It inserts a topological edge between $b_{1}$ and $b_{2}$ in order to obtain $\alpha_{2}\left(\alpha_{1}\left(b_{1}\right)\right)=\alpha_{0}\left(\alpha_{1}\left(b_{2}\right)\right)$.

The function Name_Splitted_Face, described in algorithm 2, associates the tag structures with the darts constituting $F_{1}$ and $F_{2}$. Name_Splitted_Face is used after SplitFace and it takes two darts, $b_{1}$ and $b_{2}$, incident to face $F$, as input parameters.

We show the behaviour of algorithm 2 in figure 7. Suppose that darts $b_{1}$ and $b_{2}$ are numbered 1 and 4 respectively.

The following steps are performed (figure $7 \mathrm{c}$ summarizes the whole processs):

1. Every dart of the inserted edge $A$ is processed (let $b^{\prime}$ be one of these darts). Each dart $b^{\prime}$ receives a copy of the tags related
Algo. 2 Function Name_splitted_face

Data: $b_{1}$ and $b_{2}$ : two darts representing the parameters of function SplitFace.

Result: Allocation of valid tags to the darts forming the faces $F_{1}$ and $F_{2}$.

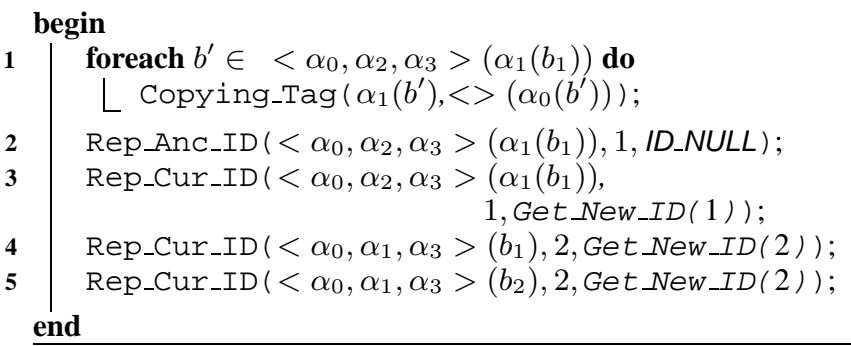

to dart $\alpha_{1}\left(b^{\prime}\right)$. In figure 7 , darts $1,3,2$ and 4 respectively propagate their tags to darts numbered $5,6,7$ and 8 .

2. Because $\mathrm{A}$ is a new edge, the edge identifier of each ancestor_tag, anc_id $d_{1}$, related to the darts belonging to $A$ is initialized with the value ID_NULL. In figure 7, darts $\{5,6,7,8\}$ have their identifier $a n c_{-} i d_{1}$ set to ID_NULL (represented by the character"-").

3. For the same reason, a new value is given to identifier cur_id $_{-} i d_{1}$ for each current_tag related to the darts forming $A$. In figure 7, identifier cur_id $_{i} d_{1}$ of the current_tags of darts $\{5,6,7,8\}$ is set to 21 .

4. We assign a new value to identifier $c u r \_i d_{2}$ for each dart belonging to $F_{1}$. In figure 7 , the value 6 is assigned to identifier cur_ $_{-} i d_{2}$ for each current_tag of the darts numbered $\{1,3,5,6,9,10\}$.

5. The same process is done on face $F_{2}$. In figure 7, identifier $c u r_{-} i d_{2}$ of each current_tag related to the darts numbered $\{2,4,7,8,11,12\}$ receives the value 10 .

Steps 2 and 3 correspond to the mechanism crea_elem applied on $A$. Indeed, these steps relate the identifier 21 characterizing $A$ to an empty set of identifiers. Steps 1, 4 and 5 implement the mechanism split_elem (see section 2.2) applied on F. The propagation (resp. the update) of tags performed during step 1 (resp. during steps 4 and 5) enables cells $F_{1}$ and $F_{2}$ to inherit the ancestor of cell $F$ : the identifier $a n c \_i d_{2} 2$ (resp. to be characterized by identifiers 6 and $10)$.

\subsection{Results}

In this section, we present the results obtained after the application of follow-up event mechanisms on the G-map model; we have successfully experimented our method on numerous models built using different operations, including classical boolean operations. The following example (see figure 8 ) has been created with boolean operations. Considering the high number of darts (872) involved in this figure, we can not show the tag structure related to each dart. Therefore, we only show some specific darts. The other darts can easily be deduced by definitions and examples introduced in sections 2.2 and 3.3.

In particular, the contents of tags (see Tab. 2a and 2b) obtained after application of the difference boolean operation (see figure 8) show that:

- Edges $A_{3}$ and $A_{4}$, respectively represented by darts $\{2.3,2.4\}$ and $\{2.9,2.10\}$, have just been created. Indeed, 


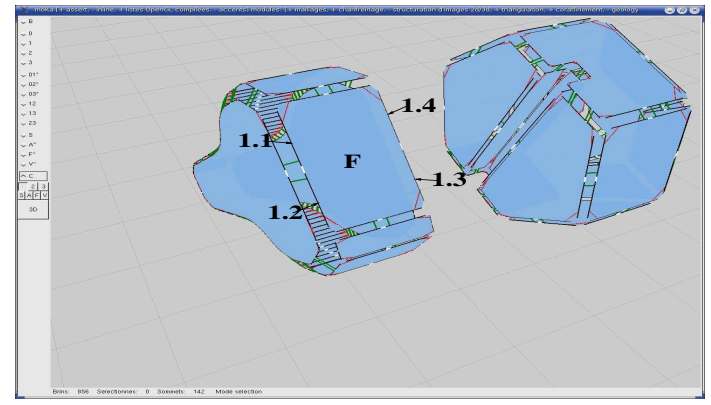

(a)

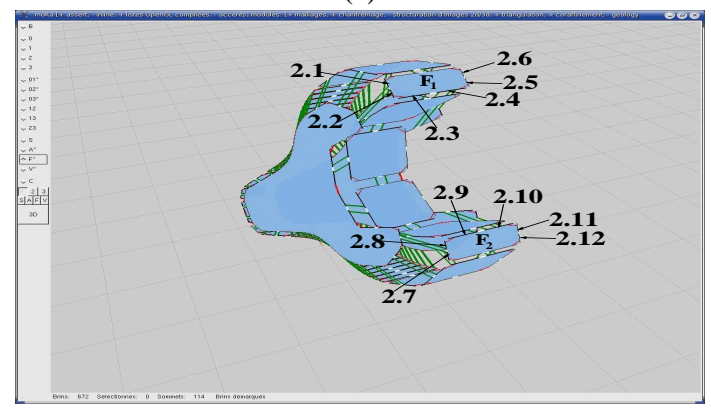

(b)

Figure 8: G-map model of spanner resulting from high level operation (boolean difference). Both figures a) and b) are Moka screenshots which show spanner in the top view. The darts are symbolized by half-edges, and involutions $\alpha_{0}$ (resp. $\left.\alpha_{1}, \alpha_{2}\right)$ are symbolized by white (resp. red, green) lines.

their identifier $a n c_{-} i d_{1}$ is equal to ID_NULL.

- Vertices $S_{1}, S_{2}, S_{3}$ and $S_{4}$, respectively represented by darts $\{2.2,2.3\},\{2.4,2.5\},\{2.8,2.9\}$ and $\{2.10,2.11\}$ (among others), have also just been created: their identifier $a n c_{-} i d_{0}$ is equal to ID_NULL.

- Face $F$, constituted by darts $\{1.1,1.2, \ldots, 1.3,1.4\}$ and characterized by the identifier cur_id $_{2} 6$ (see Tab. 2a), is split. Faces $F_{1}\left(\right.$ cur_id $\left.d_{2}=98\right)$ and $F_{2}\left(\right.$ cur_i $_{-} i d_{2}=$ 74) result from this split. Therefore, every dart being part of $F_{1}$ and $F_{2}$ (resp. $\{2.1,2.2,2.3, \ldots, 2.4,2.5,2.6\}$ and $\{2.7,2.8,2.9, \ldots, 2.10,2.11,2.12\})$ has its identifier $a n c \_i d_{2}$ equal to 6 .

- Edge $A_{1}$ (resp. edge $A_{2}$ ), constituted by darts $\{1.1,1.2\}$ (resp. $\{1.3,1.4\}$ ) and characterized by identifier cur_$_{-} i d_{1} 17$ (resp. 25), is split in edges $A_{1.1}\left(\right.$ cur_id $\left._{-} i d_{1}=197\right)$ and $A_{1.2}$ (cur_id $\left.d_{1}=133\right)$ (resp. $A_{2.1}$ and $A_{2.2}:$ cur_id $d_{1}=201$ and cur_id $\left.d_{1}=145\right)$. Therefore, darts $\{2.1,2.2,2.7,2.8\}$ forming both $A_{1.1}$ and $A_{1.2}$ have their identifier $a n c_{-} i d_{1}$ equal to 17 , and darts $\{2.5,2.6,2.11,2.12\}$, forming both $A_{2.1}$ and $A_{2.2}$, have their identifier $a n c_{-} i d_{1}$ equal to 25 .

The bulletin board (see Tab. 3), sums ups all those events. Moreover, we can indicate:

- the split of edges $A_{1}$ and $A_{2}$ (resp. of face $F$ ) characterized by identifiers $a n c_{-} i d_{1} 17$ and 25 (resp. by identifier $a n c_{-} i d_{2}$ $6)$;

- the creation of vertices (resp. edges) $S_{1}, S_{2}, S_{3}$ and $S_{4}$ (resp. $A_{3}$ and $A_{4}$ ) characterized by identifiers cur_id $d_{0} 160$, 148, 176 and 172 (resp. by identifiers cur_id $_{1} 285$ and 297);

- other events have occured during this high level operation (ta- (a) Before high level operation

\begin{tabular}{|c|c|c|}
\hline Dart & Ancestor_tag & Current_tag \\
\hline 1.1 & $(16,17,6,3)$ & $(16,17,6,3)$ \\
\hline 1.2 & $(4,17,6,3)$ & $(4,17,6,3)$ \\
\hline 1.3 & $(20,25,6,3)$ & $(20,25,6,3)$ \\
\hline 1.4 & $(24,25,6,3)$ & $(24,25,6,3)$ \\
\hline
\end{tabular}

(b) After high level operation

\begin{tabular}{|c|c|c|}
\hline Dart & Ancestor_tag & Current_tag \\
\hline 2.1 & $(16,17,6,3)$ & $(16,197,98,35)$ \\
\hline 2.2 & $(-, 17,6,3)$ & $(160,197,98,35)$ \\
\hline 2.3 & $(-,-, 6,3)$ & $(160,285,98,35)$ \\
\hline 2.4 & $(-,-, 6,3)$ & $(148,285,98,35)$ \\
\hline 2.5 & $(-, 25,6,3)$ & $(148,201,98,35)$ \\
\hline 2.6 & $(24,25,6,3)$ & $(24,201,98,35)$ \\
\hline 2.7 & $(4,17,6,3)$ & $(4,133,74,35)$ \\
\hline 2.8 & $(-, 17,6,3)$ & $(176,133,74,35)$ \\
\hline 2.9 & $(-,-, 6,3)$ & $(176,297,74,35)$ \\
\hline 2.10 & $(-,-, 6,3)$ & $(172,297,74,35)$ \\
\hline 2.11 & $(-, 25,6,3)$ & $(172,145,74,35)$ \\
\hline 2.12 & $(20,25,6,3)$ & $(20,145,74,35)$ \\
\hline
\end{tabular}

Table 2: current_tags and ancestor_tags matching the high level operation shown on figure 8 .

ble 3 only shows some of them). We can quote the deletion of vertex (resp. of edge and of face) characterized by identifiers anc_id 76 (resp. by identifiers $a n c_{-} i d_{1} 109$ and $a n c_{-} i d_{2}$ $54)$ or the creation of cells (vertex and edge) characterized by identifiers cur_id $_{i} 281$ and 160 .

Our work is currently focused on the completeness of the generated events; our method has been implemented regardless of complexity. As explained in Sections 2.2 and 2.3, there are two global traversals of the model (in order to associate and to retrieve tag structures from darts at the beginning and at the end of the high-level operation). These traversals can be optimized to process only the tag structures which have been modified by the operation.

\section{Conclusion}

In this paper, we have proposed a generic model allowing a bulletin board to be generated during a constructive operation (i.e. supplied by the API of a geometric modeling system). This bulletin board contains the complete list of events which reflects the evolution of cells of any dimension in a geometric system based on a topological model. The method uses some mechanisms for event (creation, deletion, split, merge) follow-up. More precisely, any high level operation is decomposed into a finite set of elementary operations. Each of them is associated with one or many event follow-up mechanisms. The model is generic because, on the one hand it is exclusively based on mechanisms defined independently of any geometric model, making different types of implementation possible, and, on the other hand, because these mechanisms are defined in a generic way for any dimension.

Our implementation is based on the model of generalized maps (Gmaps). By using a "tag" structure associated with each dart of a G-map, this system characterizes, through "current_tags", each cell of the current geometric model; and links through "ancestor_tags" these cells to the other cells which existed at the beginning of the high level operation. Next, the system assigns the right tag structure to each dart.

We have successfully experimented this principle on numerous models built with different operations; the co-refinement high level 


\begin{tabular}{|c|c|c|}
\hline Starting cells & Ending cells & Event type \\
\hline$\{61\}$ & $\{61\}$ & no modification \\
\hline\{\} & $\{281\}$ & edge creation \\
\hline\{\} & $\{160\}$ & vertex creation \\
\hline$\ldots$ & $\ldots$ & $\ldots$ \\
\hline\{\} & $\{\mathbf{1 4 8}\}$ & vertex creation \\
\hline\{\} & $\{\mathbf{1 6 0}\}$ & vertex creation \\
\hline\{\} & $\{\mathbf{1 7 6}\}$ & vertex creation \\
\hline\{\} & $\{\mathbf{1 7 2}\}$ & vertex creation \\
\hline$\{\mathbf{2 5}\}$ & $\{\mathbf{1 4 5 , 2 0 1}\}$ & edge split \\
\hline$\{\mathbf{1 7}\}$ & $\{\mathbf{1 3 3 , 1 9 7}\}$ & edge split \\
\hline$\{\mathbf{6}\}$ & $\{\mathbf{7 4 , 9 8}\}$ & face split \\
\hline\{\} & $\{\mathbf{2 8 5}\}$ & edge creation \\
\hline\{\} & $\{\mathbf{2 9 7}\}$ & edge creation \\
\hline$\ldots$ & $\ldots$ & .. \\
\hline$\{76\}$ & \{\} & vertex deletion \\
\hline$\{109\}$ & \{\} & edge deletion \\
\hline$\{54\}$ & \{\} & face deletion \\
\hline \multicolumn{2}{|c}{}
\end{tabular}

Table 3: Bulletin board matching the high level operation shown on figure 8.

operation is a good case study because it is complex and often used in the geometric modeling domain. The last step of the bulletin board generation process is based on the traversal of darts and the recognition of events. Our implementation enables us to realize generic mechanisms for event follow-up. It is a method useable for many and various practical application domains. It also permits the topological operations to be separated from the bulletin board generation; therefore, while topological operations are always the same for a given geometric operation, there can be several ways to generate the bulletin board, with respect to the events this bulletin board should collect.

The next step of this research is to study the possibility to test mechanisms which have been formally and independently defined in order to do a high-level experimentation and to compare the results obtained with the events contained in the bulletin board. Finally, the genericity of tag structure should allow our approach to be extended to space-time $(4 D)$ modeling for animation.

\section{References}

Armstrong, C., Bowyer, A., Cameron, S., Corney, J., Jared, G., Martin, R. R., Middleditch, A., Sabin, M., AND SAlmon, J. 2000. A Geometric Interface for Solid Modelling Specification and Report. Information Geometers Ltd.

BRISSON, E. 1993. Representing geometric structures in d dimensions: topology and order. Discrete and Computational Geometry 9, 387-426.

Brunier-Coulin, D., Pascal, D., AND MCCARThy, D. 2000. OCAF: Open Cascade Application Framework. Matra Datavision.

Gardan, Y., Minich, C., AND Perrin, E. 2003. Boolean operations on feature-based models. Journal of WSCG Winter School of Computer Graphics 1-3, 11.

GuiARD, N. 2006. Construction de modles gologiques 3D par co-raffinement de surfaces. PhD thesis, Ecole des mines.

Kitajima, K., And Yamaguchi, M. 1992. A shell oriented boolean set operations algorithm suited for the b-reps based on boundary edge loops. Systems and computers 23,6 .
KRIPAC, J. 1995. A mechanism for persistently naming topological entities in history-based parametric solid models (topological id system). Proceedings of Solid Modeling95, 21-30.

LIENHARDT, P. 1994. N-dimensional generalized combinatorial maps and cellular quasi-manifolds. International Journal of Computational Geometry and Applications 4(3), 275-324.

MA, D., AND TANG, R. 1988. Realizing the boolean operations in solid modeling technique via directed loops. Computer and Graphics 12, 3/4.

Mantyla, M., AND TAMminen, M. 1983. Localized set operations for solid modeling. Computer and Graphics 17, 3.

Marcheix, D., AND PierRa, G. 2002. A survey of the persistent naming problem. 7th ACM Symposium on Solid Modeling and Applications.

PERrin, E. 2005. Opérations booléennes: trente années d'un algorithme toujours au cœur des systèmes de CAO. Revue internationale d'ingnierie numérique 1, 265-289. 